\title{
INDUCED MAPPINGS BETWEEN QUOTIENT SPACES OF N-FOLD HYPERSPACES OF CONTINUA
}

\author{
José G. Anaya, Félix Capulín, Miguel A. Lara and Fernando \\ OROZCO-ZITLI \\ Universidad Autónoma del Estado de México, México
}

\begin{abstract}
For a continuum $X$ the hyperspace of nonempty closed subsets of $X$ with at most $n$ components is called the $n$-fold hyperspace $C_{n}(X)$ and if $m<n$ then $C_{m}(X) \subset C_{n}(X)$ so it is possible to form a quotient space $C_{n}(X) / C_{m}(X)$ identifying the set $C_{m}(X)$ to a point in $C_{n}(X)$. If $f$ is a mapping from a continuum $X$ onto a continuum $Y$ there will be a induced mappings between $C_{n}(X)$ and $C_{m}(X)$ and between the quotient spaces $C_{n}(X) / C_{m}(X)$ and $C_{n}(Y) / C_{m}(Y)$. Now if a list of function properties that are of interest to continua theorists is considered, there will be natural questions about when these properties are passed on from the functions between the continua to the induced mappings between the hyperspaces or the induced mappings between the quotients of the hyperspaces. Many of these questions have been considered extensively for the hyperspaces so the main thing that is new here is the questions and answers about the quotient spaces and their induced mappings. Here we consider the following families of mappings: atomic, atriodic, confluent, hereditarily weakly confluent, joining, light, local homeomorphism, locally confluent, locally weakly confluent, monotone, open, OM, semi-confluent and weakly confluent.
\end{abstract}

\section{INTRODUCTION}

A continuum is a nonempty, compact, connected metric space. A subcontinuum is a continuum which is a subset of a metric space. A mapping is a continuous function. The notation $\mathbb{N}$ denotes the set of all positive integers. Given a continuum $X$ and $n \in \mathbb{N}, C_{n}(X)$ denotes the hyperspace of all nonempty closed subsets of $X$ having at most $n$ components. The hyperspace $C_{n}(X)$ is considered with the Hausdorff metric, $\mathcal{H}$, (see [27, p. 1]). Let

2010 Mathematics Subject Classification. 54C05, 54C10, 54B20, 54B15.

Key words and phrases. Continuum, hyperspace, induced mapping, quotient space. 
$n, m \in \mathbb{N}$ be such that $n>m$. The notation $S C_{m}^{n}(X)$ denotes the quotient space $C_{n}(X) / C_{m}(X)$ obtained of $C_{n}(X)$ identifying the set $C_{m}(X)$ to a point. And the function $\rho_{X}^{n, m}: C_{n}(X) \rightarrow S C_{m}^{n}(X)$ denotes the quotient mapping.

Given a mapping $f: X \rightarrow Y$ between continua, the mapping $C_{n}(f)$ : $C_{n}(X) \rightarrow C_{n}(Y)$ given by $C_{n}(f)(A)=f(A)$ is a mapping induced by $f$. Let $S C_{m}^{n}(f): S C_{m}^{n}(X) \rightarrow S C_{m}^{n}(Y)$ be a function defined such a way that

$$
S C_{m}^{n}(f)\left(\rho_{X}^{n, m}(A)\right)=\rho_{Y}^{n, m}(f(A)) .
$$

By [15, Theorem 4.3], $S C_{m}^{n}(f)$ is a continuous function. Moreover the following diagram is commutative.

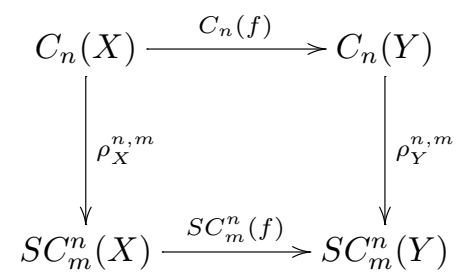

Let $\mathcal{M}$ be a class of mappings between continua. A general problem in Continuum Theory is to determine all possible relationships among the following statements:

(1) $f \in \mathcal{M}$

(2) $C_{n}(f) \in \mathcal{M}$;

(3) $S C_{m}^{n}(f) \in \mathcal{M}$.

Readers especially interested in this topic are referred to [1]-[14], [16]-[21].

In this paper we study the interrelations among the statements (1)-(3), where $\mathcal{M}$ is the family of: atomic, atriodic, confluent, hereditarily weakly confluent, joining, light, local homeomorphism, locally confluent, locally weakly confluent, monotone, open, OM, semi-confluent or weakly confluent mappings.

\section{Preliminaries}

Let $X$ be a continuum with metric $d$. Let $\epsilon>0$ and $A \subset X$, we define $\mathcal{V}_{\epsilon}^{d}(A)=\{x \in X$ : there exists $y \in A$ such that $d(x, y)<\epsilon\}$, and we use the symbol $\mathrm{Cl}(\mathrm{A})$ to denote the closure of $A$ in $X$. An order arc in $C_{n}(X)$ is an $\operatorname{arc} \alpha:[0,1] \rightarrow C_{n}(X)$ such that if $0 \leq s<t \leq 1$, then $\alpha(s) \subset \alpha(t)$ and $\alpha(s) \neq$ $\alpha(t)$. Given a finite collection $K_{1}, \ldots, K_{r}$ of subsets of $X,\left\langle K_{1}, \ldots, K_{r}\right\rangle$, is used to denote the following subset of $C_{n}(X)$,

$$
\left\{A \in C_{n}(X): A \subset \bigcup_{i=1}^{r} K_{i}, A \cap K_{i} \neq \emptyset \text { for each } i \in\{1, \ldots, r\}\right\} .
$$

It is well known that the family of all subsets of $C_{n}(X)$ of the form $\left\langle K_{1}, \ldots, K_{r}\right\rangle$, where each $K_{i}$ is an open subset of $X$, forms a base for a topology for $C_{n}(X)$ (see [27, Theorem 0.11]) called the Vietoris topology, which 
coincide with the topology induced on $C_{n}(X)$ by the Hausdorff metric (see [27, Theorem 0.13]).

Given two positive integers $s>k$, we define $\mathcal{C}_{k}^{s}(X)=C_{s}(X) \backslash C_{k}(X)$. Notice that $\left.\rho_{X}^{s, k}\right|_{\mathcal{C}_{k}^{s}(X)}: \mathcal{C}_{k}^{s}(X) \rightarrow S C_{k}^{s}(X) \backslash \rho_{X}^{s, k}\left(C_{k}(X)\right)$ is a homeomorphism.

A surjective mapping $f: X \rightarrow Y$ between continua is:

- atriodic if, for each subcontinuum $Q$ of $Y$, there are two components $C_{1}$ and $C_{2}$ of $f^{-1}(Q)$ such that $f\left(C_{1}\right) \cup f\left(C_{1}\right)=Q$ and for each component $C$ of $f^{-1}(Q)$, we have either $f(C)=Q$ or $f(C) \subset f\left(C_{1}\right)$ or $f(C) \subset f\left(C_{2}\right)$;

- atomic if, for each subcontinuum $K$ of $X$ such that $f(K)$ is nondegenerate, $f^{-1}(f(K))=K$;

- confluent if, for every subcontinuum $K$ of $Y$ and for each component $M$ of $f^{-1}(K), f(M)=K$;

- joining if, for every subcontinuum $B$ of $Y$ and every pair of components $C$ and $D$ of $f^{-1}(B), f(C) \cap f(D) \neq \emptyset$;

- light if $f^{-1}(y)$ is totally disconnected for each $y \in Y$;

- a local homeomorphism if, for each point $x \in X$, there exists an open neighbourhood $U$ of $x$ in $X$ such that $f(U)$ is a open neighbourhood of $f(x)$ in $Y$ and the partial mapping $\left.f\right|_{U}: U \rightarrow f(U)$ is a homeomorphism;

- monotone if $f^{-1}(y)$ is connected for each $y \in Y$;

- open if $f(U)$ is open in $Y$ for each open subset $U$ of $X$;

- an OM-mapping if there exist a continuum $Z$ and mappings $g: X \rightarrow Z$ and $h: Z \rightarrow Y$ such that $f=h \circ g, g$ is monotone and $h$ is open;

- semi-confluent if, for every subcontinuum $B$ of $Y$ and every pair of components $C$ and $D$ of $f^{-1}(B)$, either $f(C) \subset f(D)$ or $f(D) \subset f(C)$;

- weakly confluent if, for each subcontinuum $K$ of $Y$, there exists a subcontinuum $M$ of $X$ such that $f(M)=K$;

- locally confluent(locally weakly confluent) provided for each point $y \in$ $Y$, there exists a closed neighbourhood $F$ of $y$ in $Y$ such that the partial mapping $\left.f\right|_{f^{-1}(F)}$ is a confluent mapping(a weakly confluent mapping) of $f^{-1}(F)$ onto $F$;

- hereditarily weakly confluent if, for each nondegenerate subcontinuum $M$ of $X,\left.f\right|_{M}$ is weakly confluent.

As a consequence of the fact that $C_{n}(X)$ is arcwise connected [23, Theorem $3.1]$ and [28, Theorem 3.10] we have the following proposition.

Proposition 2.1. Let $X$ be a continuum and $n \geq 2$. Then $S C_{m}^{n}(X)$ is an arcwise connected continuum.

The next theorem is proved in [23, Theorem 3.3].

Proposition 2.2. Let $X$ be a continuum, if $n \geq 2$ then $\mathcal{C}_{n-1}^{n}(X)$ is dense on $C_{n}(X)$. 
Lemma 2.3. Let $X$ be a continuum and $n \geq 2$. If $\mathcal{U} \subset C_{n}(X)$ such that $\mathcal{U} \cap C_{m}(X) \neq \emptyset$ and $\rho_{X}^{n, m}(\mathcal{U})$ is an open subset of $S C_{m}^{n}(X)$, then $C_{m}(X) \subset$ $\mathrm{Cl}(\mathcal{U})$.

Proof. Let $A \in C_{m}(X)$ such that $A \notin \mathrm{Cl}(\mathcal{U})$. Then there is an $\epsilon>0$ such that $\mathcal{V}_{\epsilon}^{\mathcal{H}}(A) \cap \mathcal{U}=\emptyset$. Thus, since $\left(\rho_{X}^{n, m}\right)^{-1}\left(\rho_{X}^{n, m}(\mathcal{U})\right)=\mathcal{U} \cup C_{m}(X)$ is an open subset of $C_{n}(X)$, there exists $0<\delta \leq \epsilon$ such that $\mathcal{V}_{\delta}^{\mathcal{H}}(A) \subset C_{m}(X)$. This contradicts Proposition 2.2.

Proposition 2.4. Let $f: X \rightarrow Y$ be a surjective mapping between continua and $1 \leq r \leq n$. Let $K_{1}, \ldots, K_{r}$ be nonempty disjoint closed subsets of $Y$. For each $i \in\{1, \ldots, r\}$, let $M_{i}$ be a component of $f^{-1}\left(K_{i}\right)$. Then

(1) $\left\langle M_{1}, \ldots, M_{r}\right\rangle$ is a component of $C_{n}(f)^{-1}\left(\left\langle K_{1}, \ldots, K_{r}\right\rangle\right)$.

(2) If $M$ is a component of $f^{-1}\left(K_{i}\right)$ such that $M \neq M_{i}$ and $r<n$, then $\left\langle M_{1}, \ldots, M_{r}, M\right\rangle$ is a component of $C_{n}(f)^{-1}\left(\left\langle K_{1}, \ldots, K_{r}\right\rangle\right)$.

(3) If $r>m$, then $\rho_{X}^{n, m}\left(\left\langle M_{1}, \ldots, M_{r}\right\rangle\right)$ is a component of

$$
S C_{m}^{n}(f)^{-1}\left(\rho_{Y}^{n, m}\left(\left\langle K_{1}, \ldots, K_{r}\right\rangle\right)\right) .
$$

Proof. We will prove (1). By [2, Proposition 2.3$],\left\langle M_{1}, \ldots, M_{r}\right\rangle$ is connected. Note that $\left\langle M_{1}, \ldots, M_{r}\right\rangle \subset C_{n}(f)^{-1}\left(\left\langle K_{1}, \ldots, K_{r}\right\rangle\right)$. Consider $\mathcal{C}$, the component of $C_{n}(f)^{-1}\left(\left\langle K_{1}, \ldots, K_{r}\right\rangle\right)$ containing $\left\langle M_{1}, \ldots, M_{r}\right\rangle$. Clearly

$$
\bigcup_{i=1}^{r} M_{i} \subset \bigcup \mathcal{C} \subset \bigcup_{i=1}^{r} f^{-1}\left(K_{i}\right) .
$$

Now, let $P$ be a component of $\bigcup \mathcal{C}$. By [20, Lemma 3.1], $P \cap M_{j} \neq \emptyset$ for some $j \in\{1, \ldots, r\}$. Thus, $P=M_{j}$. Hence, using [20, Lemma 3.1], $\mathcal{C}=$ $\left\langle M_{1}, \ldots, M_{r}\right\rangle$.

The proof of (2) is similar to the proof of $(1)$.

To prove (3), consider $\mathcal{D}$ the component of $S C_{m}^{n}(f)^{-1}\left(\rho_{Y}^{n, m}\left(\left\langle K_{1}, \ldots, K_{r}\right\rangle\right)\right)$ containing $\rho_{X}^{n, m}\left(\left\langle M_{1}, \ldots, M_{n}\right\rangle\right)$. Clearly, $\left\langle M_{1}, \ldots, M_{n}\right\rangle \subset\left(\rho_{X}^{n, m}\right)^{-1}(\mathcal{D})$. We need to prove that $\left(\rho_{X}^{n, m}\right)^{-1}(\mathcal{D}) \subset C_{n}(f)^{-1}\left(\left\langle K_{1}, \ldots, K_{r}\right\rangle\right)$. Notice that $S C_{m}^{n}(f)\left(\rho_{X}^{n, m}(A)\right)=\rho_{Y}^{n, m}\left(C_{n}(f)(A)\right)$ for each $A \in \mathcal{C}_{m}^{n}(X)$. Since $\rho_{Y}^{n, m}\left(C_{m}(Y)\right) \notin \rho_{Y}^{n, m}\left(\left\langle K_{1}, \ldots, K_{r}\right\rangle\right), \quad \rho_{X}^{n, m}\left(C_{m}(X)\right) \notin \mathcal{D}$ and $C_{m}(X) \cap$ $\left(\rho_{X}^{n, m}\right)^{-1}(\mathcal{D})=\emptyset$. Then, $\rho_{Y}^{n, m}\left(C_{n}(f)\left(\left(\rho_{X}^{n, m}\right)^{-1}(\mathcal{D})\right)\right) \subset \rho_{Y}^{n, m}\left(\left\langle K_{1}, \ldots, K_{r}\right\rangle\right)$. Thus $C_{n}(f)\left(\left(\rho_{X}^{n, m}\right)^{-1}(\mathcal{D})\right) \subset\left\langle K_{1}, \ldots, K_{r}\right\rangle$. Now, since $\rho_{X}^{n, m}$ is monotone, $\left(\rho_{X}^{n, m}\right)^{-1}(\mathcal{D}) \subset\left\langle M_{1}, \ldots, M_{n}\right\rangle, \mathcal{D}=\rho_{X}^{n, m}\left(\left\langle M_{1}, \ldots, M_{n}\right\rangle\right)$.

\section{HOMEOMORPHISMS AND OPEN MAPPINGS}

TheOREM 3.1. Let $f: X \rightarrow Y$ be a mapping between continua and $n \geq 2$. Then the following conditions are equivalent.

(1) $f$ is a homeomorphism.

(2) $C_{n}(f)$ is a homeomorphism.

(3) $S C_{m}^{n}(f)$ is a homeomorphism. 
Proof. $(1) \Rightarrow(2)$. It follows from [12, Theorem 46].

It is easy to see $(2) \Rightarrow(3)$.

In order to prove that $(3) \Rightarrow(1)$, let $y \in Y$ and let $y_{1}, \ldots, y_{m} \in Y \backslash\{y\}$ such that $y_{i} \neq y_{j}$ if $i \neq j$. Let $B=\left\{y_{1}, \ldots, y_{m}, y\right\} \in \mathcal{C}_{m}^{n}(Y)$. Since $S C_{m}^{n}(f)$ is a homeomorphism, there is $A \in \mathcal{C}_{m}^{n}(X)$ such that $S C_{m}^{n}(f)\left(\rho_{X}^{n, m}(A)\right)=$ $\rho_{Y}^{n, m}(B)$. So, $C_{n}(f)(A)=B=f(A)$. Thus, $f$ is surjective. Now, let $x, y \in X$ such that $x \neq y$ and let $z_{1}, \ldots, z_{m} \in X \backslash\{x, y\}$ such that $A=\left\{z_{1}, \ldots, z_{m}, x\right\}, B=\left\{z_{1}, \ldots, z_{m}, y\right\} \in \mathcal{C}_{m}^{n}(X)$. Since $S C_{m}^{n}(f)$ is a homeomorphism, $\rho_{Y}^{n, m}(f(A)) \neq \rho_{Y}^{n, m}(f(B))$. So $f(x) \neq f(y)$. Therefore $f$ is a homeomorphism.

Theorem 3.2. Let $f: X \rightarrow Y$ be a mapping between continua and $n \geq 2$. Then the following conditions are equivalent.

(1) $f$ is homeomorphism.

(2) $C_{n}(f)$ is open.

(3) $S C_{m}^{n}(f)$ is open for every $1 \leq m<n$.

Proof. By [1, Corollary 3.3], (1) and (2) are equivalent.

$(1) \Rightarrow(3)$ Follows from Theorem 3.1. To prove $(3) \Rightarrow(1)$, let $x_{1}, x_{2} \in X$ such that $x_{1} \neq x_{2}$ and $f\left(x_{1}\right)=f\left(x_{2}\right)$. Consider $A \in \mathcal{C}_{m}^{m+1}(X)$ such that $\left\{x_{1}\right\}$ and $\left\{x_{2}\right\}$ are components of $A$. Choose $\delta>0$ such that $\mathcal{V}_{\delta}^{\mathcal{H}}(A) \bigcap C_{m}(X)=\emptyset$. Let $0<\epsilon<\delta$. Then, $\rho_{X}^{n, m}\left(\mathcal{V}_{\epsilon}^{\mathcal{H}}(A)\right)$ is an open subset of $S C_{m}^{n}(X)$. Since $S C_{m}^{n}(f)\left(\rho_{X}^{n, m}\left(\mathcal{V}_{\epsilon}^{\mathcal{H}}(A)\right)\right)=\rho_{Y}^{n, m}\left(C_{n}(f)\left(\mathcal{V}_{\epsilon}^{\mathcal{H}}(A)\right)\right), \rho_{Y}^{n, m}\left(C_{n}(f)\left(\mathcal{V}_{\epsilon}^{\mathcal{H}}(A)\right)\right)$ is open in $S C_{m}^{n}(Y)$. By Lemma 2.3, $C_{m}(Y) \subset \bigcap_{\epsilon>0} \mathrm{Cl}\left(C_{n}(f)\left(\mathcal{V}_{\epsilon}^{\mathcal{H}}(A)\right)\right)$. Thus, $C_{m}(Y)=\left\{C_{n}(f)(A)\right\}$, a contradiction. Therefore $f$ is a homeomorphism.

Concerning the last theorem, we can see in the following example that there exists an open mapping between continua such that $S C_{m}^{n}(f)$ is not open, in other words, the condition that $f$ is homeomorphism is essential to prove that $S C_{m}^{n}(f)$ is an open mapping.

ExAmple 3.3. There exist continua $X$ and $Y$, and an open mapping $f: X \rightarrow Y$ such that $S C_{m}^{n}(f)$ is not open for $n \geq 2$.

Consider the mapping $f:[-1,1] \rightarrow[0,1]$ defined by $f(x)=|x|$. Clearly, $f$ is open. By Theorem 3.2, $C_{n}(f)$ and $S C_{m}^{n}(f)$ are not open for $n \geq 2$.

\section{Monotone mappings}

TheOREM 4.1. Let $f: X \rightarrow Y$ be a mapping between continua and $n \geq 2$. Then the following conditions are equivalent.

(1) $f$ is monotone.

(2) $C_{n}(f)$ is monotone.

(3) $S C_{m}^{n}(f)$ is monotone for every $1 \leq m<n$. 
Proof. By [12, Theorem 4], (1) and (2) are equivalent.

To prove that $(2) \Rightarrow(3)$, let $B \in C_{n}(Y)$. Since $C_{n}(f)$ and $\rho_{X}^{n, m}$ are monotone mappings and

$$
\rho_{X}^{n, m}\left(C_{n}(f)^{-1}\left(\left(\rho_{Y}^{n, m}\right)^{-1}\left(\rho_{Y}^{n, m}(B)\right)\right)\right)=S C_{m}^{n}(f)^{-1}\left(\rho_{Y}^{n, m}(B)\right),
$$

$S C_{m}^{n}(f)^{-1}\left(\rho_{Y}^{n, m}(B)\right)$ is connected. Hence, $S C_{m}^{n}(f)$ is monotone.

Now, we show that $(3) \Rightarrow(1)$. Suppose that there exists $y \in Y$ such that $f^{-1}(y)$ is not connected. Let $K_{1}$ and $K_{2}$ be different components of $f^{-1}(y)$. Consider $\left\{z_{1}, \ldots, z_{n-1}\right\} \in Y \backslash\{y\}$ such that $z_{i} \neq z_{j}$ if $i \neq j$. Let $M_{i}$ be a component of $f^{-1}\left(z_{i}\right)$, for each $i \in\{1, \ldots, n-$ 1\}. By Proposition 2.4, $\rho_{X}^{n, m}\left(\left\langle M_{1}, \ldots, M_{n-1}, K_{i}\right\rangle\right)$ is a component of $S C_{m}^{n}(f)^{-1}\left(\rho_{Y}^{n, m}\left(\left\{z_{1}, \ldots, z_{n-1}, y\right\}\right)\right)$, for each $i \in\{1,2\}$. Since $S C_{m}^{n}(f)$ is a monotone mapping, $S C_{m}^{n}(f)^{-1}\left(\rho_{Y}^{n, m}\left(\left\{z_{1}, \ldots, z_{n-1}, y\right\}\right)\right)$ is connected. Thus, $\rho_{X}^{n, m}\left(\left\langle M_{1}, \ldots, M_{n-1}, K_{1}\right\rangle\right)=\rho_{X}^{n, m}\left(\left\langle M_{1}, \ldots, M_{n-1}, K_{2}\right\rangle\right)$. Then, $K_{1}=K_{2}$. A contradiction.

\section{Confluent mappings}

Proposition 5.1. Let $f: X \rightarrow Y$ be a mapping between continua.

(1) If $S C_{m}^{n}(f)$ is confluent, then for each subcontinuum $\mathcal{B} \subset \mathcal{C}_{m}^{n}(Y)$ and each component $\mathcal{A}$ of $C_{n}(f)^{-1}(\mathcal{B}), C_{n}(f)(\mathcal{A})=\mathcal{B}$.

(2) If $S C_{m}^{n}(f)$ is weakly confluent, then for each subcontinuum $\mathcal{B} \subset \mathcal{C}_{m}^{n}(Y)$, there exists $\mathcal{A}$ be a subcontinuum of $C_{n}(X)$ such that $C_{n}(f)(\mathcal{A})=\mathcal{B}$.

Proof. It follows of the facts that $\left.\rho_{X}^{n, m}\right|_{\mathcal{C}_{m}^{n}(X)}$ and $\left.\rho_{Y}^{n, m}\right|_{\mathcal{C}_{m}^{n}(Y)}$ are homeomorphisms and

$$
\left.C_{n}(f)\right|_{C_{n}(f)^{-1}\left(\mathcal{C}_{m}^{n}(Y)\right)}=\left.\left.\left(\rho_{Y}^{n, m}\right)^{-1}\right|_{\mathcal{C}_{m}^{n}(Y)} \circ S C_{m}^{n}(f) \circ \rho_{X}^{n, m}\right|_{\mathcal{C}_{m}^{n}(X)} .
$$

TheOREM 5.2. Let $f: X \rightarrow Y$ be a mapping between continua and let $n \geq 2$. We consider the following conditions:

(1) $f$ is confluent;

(2) $C_{n}(f)$ is confluent;

(3) $S C_{m}^{n}(f)$ is confluent for all $m<n$.

Then condition (2) implies (3) and each of the conditions (2) and (3) implies (1).

Proof. $(2) \Rightarrow(3)$. Since $C_{n}(f)$ is confluent, $\rho_{Y}^{n, m}$ is monotone and $\rho_{Y}^{n, m} \circ C_{n}(f)=S C_{m}^{n}(f) \circ \rho_{X}^{n, m}$, by 5.4 of $\left[24\right.$, p. 29], $S C_{m}^{n}(f) \circ \rho_{X}^{n, m}$ is confluent. Hence by 5.16 of [24, p. 32], $S C_{m}^{n}(f)$ is confluent.

We will prove that $(3) \Rightarrow(1)$. Let $B$ be a proper subcontinuum of $Y$ and $K$ be a component of $f^{-1}(B)$. Let $\left\{z_{1}, \ldots, z_{m}\right\} \subset Y \backslash B$ such that $z_{i} \neq z_{j}$ if $i \neq j$. Let $M_{i}$ be a component of $f^{-1}\left(z_{i}\right)$, for each $i \in\{1, \ldots, m\}$. By Proposition $2.4,\left\langle M_{1}, \ldots, M_{m}, K\right\rangle$ is a component of $C_{n}(f)^{-1}\left(\left\langle\left\{z_{1}\right\}, \ldots,\left\{z_{m}\right\}, B\right\rangle\right)$. 
Notice that $\left\langle\left\{z_{1}\right\}, \ldots,\left\{z_{m}\right\}, B\right\rangle \subset \mathcal{C}_{m}^{n}(Y)$. Then, by the Proposition 5.1, $C_{n}(f)\left(\left\langle M_{1}, \ldots, M_{m}, K\right\rangle\right)=\left\langle\left\{z_{1}\right\}, \ldots,\left\{z_{m}\right\}, B\right\rangle$. Therefore $f(K)=B$.

Clearly $(2) \Rightarrow(1)$.

Theorem 5.3. Let $f: X \rightarrow Y$ be a mapping between continua and let $n \geq 4$. Then the following conditions are equivalent.

1. $f$ is a monotone mapping.

2. $S C_{m}^{n}(f)$ is a confluent mapping for $2 \leq m \leq n-2$.

Proof. (1) $\Rightarrow(2)$. It follows from Theorem 4.1 and (3.2) of [24, p. 13].

To prove that $f$ is a monotone mapping, suppose to the contrary, that is, there exists $y \in Y$ such that $f^{-1}(y)$ is not connected. Let $M_{0}$ and $M_{1}$ be different components of $f^{-1}(y)$. By [28, Corollary 5.5], there exists a subcontinuum $K$ of $Y$ such that $y \notin K$. Take $\left\{z_{2}, \ldots, z_{m}\right\} \subset Y \backslash(K \cup\{y\})$ such that $z_{i} \neq z_{j}$ if $i \neq j$. Let $M_{i}$ be a component of $f^{-1}\left(z_{i}\right)$, for each $i \in\{2, \ldots, m\}$ and let $M_{m+1}$ be a component of $f^{-1}(K)$. Notice that $\left\langle\{y\},\left\{z_{2}\right\}, \ldots,\left\{z_{m}\right\}, K\right\rangle \subset$ $\mathcal{C}_{m}^{n}(Y)$ and $\mathcal{C}_{n-1}^{n}(Y) \cap\left\langle\{y\},\left\{z_{2}\right\}, \ldots,\left\{z_{m}\right\}, K\right\rangle \neq \emptyset$. By Proposition 2.4, $\left\langle M_{0}, \ldots, M_{m+1}\right\rangle$ is a component of $C_{n}(f)^{-1}\left(\left\langle\{y\},\left\{z_{2}\right\}, \ldots,\left\{z_{m}\right\}, K\right\rangle\right)$. By Proposition 5.1, $C_{n}(f)\left(\left\langle M_{0}, \ldots, M_{m+1}\right\rangle\right)=\left\langle\{y\},\left\{z_{2}\right\}, \ldots,\left\{z_{m}\right\}, K\right\rangle$. Thus $\left\langle\{y\},\left\{z_{2}\right\}, \ldots,\left\{z_{m}\right\}, K\right\rangle \subset C_{n-1}(Y)$. A contradiction.

The next example shows that (1) does not imply (3) in Theorem 5.2.

ExAmPLE 5.4. There exist continua $X$ and $Y$, and a confluent mapping $f: X \rightarrow Y$ such that $S C_{m}^{n}(f)$ is not confluent for $n \geq 4$.

Consider the mapping $f:[-1,1] \rightarrow[0,1]$ defined by $f(x)=|x|$. Clearly, $f$ is confluent but not monotone. By Theorem 5.3, $S C_{m}^{n}(f)$ is not confluent for $n \geq 4$ and $2 \leq m \leq n-2$.

Theorem 5.5. Let $f: X \rightarrow Y$ be a mapping between continua and let $n \geq 2$. Consider the following conditions:

(1) $f$ is weakly confluent;

(2) $C_{n}(f)$ is weakly confluent;

(3) $S C_{m}^{n}(f)$ is weakly confluent.

Then condition (2) implies (3) and each of the conditions (2) and (3) implies (1).

Proof. We will prove that $(2) \Rightarrow(3)$. Since $C_{n}(f)$ is weakly confluent, $\rho_{Y}^{n, m}$ is monotone and $\rho_{Y}^{n, m} \circ C_{n}(f)=S C_{m}^{n}(f) \circ \rho_{X}^{n, m}$, by (5.4) of [24, p. 29], $S C_{m}^{n}(f) \circ \rho_{X}^{n, m}$ is weakly confluent. By 5.16 of $\left[24\right.$, p. 32], $S C_{m}^{n}(f)$ is weakly confluent.

Now, we will show that $(3) \Rightarrow(1)$. Let $B$ be a proper subcontinuum of $Y$. Consider $\left\{z_{1}, \ldots, z_{n-1}\right\} \subset Y \backslash B$ such that $z_{i} \neq z_{j}$ if $i \neq j$. Note that $\left\langle\left\{z_{1}\right\}, \ldots,\left\{z_{n-1}\right\}, B\right\rangle \subset \mathcal{C}_{n-1}^{n}(Y)$. Then, by Proposition 5.1, there exists a component $\mathcal{K}$ of $C_{n}(f)^{-1}\left(\left\langle\left\{z_{1}\right\}, \ldots,\left\{z_{n-1}\right\}, B\right\rangle\right)$ such that $C_{n}(f)(\mathcal{K})=$ 
$\left\langle\left\{z_{1}\right\}, \ldots,\left\{z_{n-1}\right\}, B\right\rangle$. Since $\mathcal{K} \subset \mathcal{C}_{n-1}^{n}(X)$, we can find subset $M_{1}, \ldots, M_{n}$ of $X$, such that $M_{i}$ is a component of $f^{-1}\left(z_{i}\right)$, for each $i \in\{1, \ldots, n-1\}, M_{n}$ is a component of $f^{-1}(B)$ and $\left\langle M_{1}, \ldots, M_{n}\right\rangle \cap \mathcal{K} \neq \emptyset$. Now, by Proposition $2.4,\left\langle M_{1}, \ldots, M_{n}\right\rangle$ is a component of $C_{n}(f)^{-1}\left(\left\langle\left\{z_{1}\right\}, \ldots,\left\{z_{n-1}\right\}, B\right\rangle\right)$. Thus $\mathcal{K}=\left\langle M_{1}, \ldots, M_{n}\right\rangle$. Hence $f\left(M_{n}\right)=B$.

Clearly $(2) \Rightarrow(1)$.

ThEOREM 5.6. Let $f: X \rightarrow Y$ be a mapping between continua and let $n \geq 2$. Consider the following conditions:

(1) $f$ is hereditarily weakly confluent;

(2) $C_{n}(f)$ is hereditarily weakly confluent;

(3) $S C_{m}^{n}(f)$ is hereditarily weakly confluent.

Then condition (2) implies (3) and each of the conditions (2) and (3) implies (1).

Proof. $(2) \Rightarrow(3)$. By [12, Theorem 44], $f$ is a homeomorphism. Then, by Theorem 3.1, $S C_{m}^{n}(f)$ is a homeomorphism. Thus $S C_{m}^{n}(f)$ is hereditarily weakly confluent.

Now we will prove $(3) \Rightarrow(1)$. Let $D$ be a subcontinuum of $X$, consider two cases, $f(D)=Y$ or $f(Y) \neq X$. Suppose $f(D)=Y$. Since $\left.S C_{m}^{n}(f)\right|_{S C_{m}^{n}(D)}$ is weakly confluent, by Theorem $5.5,\left.f\right|_{D}$ is weakly confluent. Now, suppose that $f(D)$ is a subcontinuum proper of $Y$. Let $B$ be a subcontinuum of $f(D)$. Consider $\left\{z_{1}, \ldots, z_{n-1}\right\} \subset Y \backslash B$ such that $z_{i} \neq z_{j}$ if $i \neq j$. Let $M_{i}$ be a component of $f^{-1}\left(z_{i}\right)$, for each $i \in\{1, \ldots, n-1\}$. Notice that $\mathcal{D}=$ $\rho_{X}^{n, m}\left(\left\langle M_{1}, \ldots, M_{n-1}, D\right\rangle\right)$ is a subcontinuum of $S C_{m}^{n}(X)$. Since $\left.S C_{m}^{n}(f)\right|_{\mathcal{D}}$ is weakly confluent and $\rho_{Y}^{n, m}\left(\left\langle\left\{z_{1}\right\}, \ldots,\left\{z_{n-1}\right\}, B\right\rangle\right)$ is a subcontinnum of $S C_{m}^{n}(f)(\mathcal{D})$, there exists a subcontinuum $\mathcal{W}$ of $\mathcal{D}$ such that $S C_{m}^{n}(f)(\mathcal{W})=$ $\rho_{Y}^{n, m}\left(\left\langle\left\{z_{1}\right\}, \ldots,\left\{z_{n-1}\right\}, B\right\rangle\right)$. Moreover, $\left(\rho_{X}^{n, m}\right)^{-1}(\mathcal{W})$ is a subcontinuum of $\left\langle M_{1}, \ldots, M_{n-1}, D\right\rangle$ such that $C_{n}(f)\left(\left(\rho_{X}^{n, m}\right)^{-1}(\mathcal{W})\right)=\left\langle\left\{z_{1}\right\}, \ldots,\left\{z_{n-1}\right\}, B\right\rangle$. By [20, Lemma 3.1], $K=\bigcup\left(\rho_{X}^{n, m}\right)^{-1}(\mathcal{W}) \cap D$ is a nonempty connected set. Thus $f(K)=B$.

TheOREM 5.7. Let $f: X \rightarrow Y$ be a mapping between continua and $n \geq 2$. Consider the following conditions:

(1) $f$ is semi-confluent;

(2) $C_{n}(f)$ is semi-confluent;

(3) $S C_{m}^{n}(f)$ is semi-confluent.

Then (2) implies (3) and each of the conditions (2) and (3) implies (1).

Proof. $(2) \Rightarrow(3)$. Since $\rho_{Y}^{n, m}$ is monotone, by Statement 1.1 of $[10$, p. 41], $\rho_{Y}^{n, m} \circ C_{n}(f)$ is semi-confluent. Thus $S C_{m}^{n}(f) \circ \rho_{X}^{n, m}$ is semi-confluent. By 5.16 of [24, p. 32], $S C_{m}^{n}(f)$ is semi-confluent.

We will prove that $(3) \Rightarrow(1)$. Let $B$ be a proper subcontinuum of $Y$. Let $C_{1}$ and $C_{2}$ are components of $f^{-1}(B)$. Consider $\left\{z_{1}, \ldots, z_{m}\right\} \subset Y \backslash B$ 
such that $z_{i} \neq z_{j}$ if $i \neq j$. Let $M_{i}$ be a component of $f^{-1}\left(z_{i}\right)$, for each $i \in\{1, \ldots, m\}$. By Proposition 2.4, $\rho_{X}^{n, m}\left(\left\langle M_{1}, \ldots, M_{m}, C_{i}\right\rangle\right)$ is a component of $S C_{m}^{n}(f)^{-1}\left(\rho_{Y}^{n, m}\left(\left\langle\left\{z_{1}\right\}, \ldots,\left\{z_{m}\right\}, B\right\rangle\right)\right)$, for each $i \in\{1,2\}$. Without of lost generality we can suppose that $S C_{m}^{n}(f)\left(\rho_{X}^{n, m}\left(\left\langle M_{1}, \ldots, M_{m}, C_{1}\right\rangle\right)\right) \subset$ $S C_{m}^{n}(f)\left(\rho_{X}^{n, m}\left(\left\langle M_{1}, \ldots, M_{m}, C_{2}\right\rangle\right)\right)$. Thus

$$
C_{n}(f)\left(\left\langle M_{1}, \ldots, M_{m}, C_{1}\right\rangle\right) \subset C_{n}(f)\left(\left\langle M_{1}, \ldots, M_{m}, C_{2}\right\rangle\right) .
$$

Hence $f\left(C_{1}\right) \subset f\left(C_{2}\right)$.

$(2) \Rightarrow(1)$ is obvious.

\section{OM MAPPINGS}

In this part we use the following definition to limit superior of a sequence of subsets

Definition 6.1. Let $X$ be a continuum. Given a sequence $\left\{A_{m}\right\}_{m \in \mathbb{N}}$ of subsets of $X$ we define $\lim \sup _{m \rightarrow \infty} A_{m}$ as the set of points $x \in X$ such that there exists a sequence of positive numbers $m_{1}<m_{2}<\cdots$ and points $x_{m_{k}} \in A_{m_{k}}$ such that $\lim x_{m_{k}}=x$.

The following characterization of $O M$ mappings was showed in [12, p. 788].

Lemma 6.2. A mapping $f: X \rightarrow Y$ between continua is an OM-mapping if and only if, for each point $y \in Y$ and each sequence of points $y_{m} \in Y$ tending to $y$ the set $\limsup _{m \rightarrow \infty} f^{-1}\left(y_{m}\right)$ meets each component of $f^{-1}(y)$.

Theorem 6.3. Let $f: X \rightarrow Y$ be a mapping between continua and let $n \geq 2$. Consider the following conditions:

(1) $f$ is an OM-mapping;

(2) $C_{n}(f)$ is an OM-mapping;

(3) $S C_{m}^{n}(f)$ is an OM-mapping.

Then condition (2) implies (3) and each of the conditions (2) and (3) implies (1).

Proof. $(2) \Rightarrow(3)$. Since $C_{n}(f)$ is an $O M$-mapping, $\rho_{Y}^{n, m}$ is monotone and $\rho_{Y}^{n, m} \circ C_{n}(f)=S C_{m}^{n}(f) \circ \rho_{X}^{n, m}$, by 5.4 of [24, p. 28], $S C_{m}^{n}(f) \circ \rho_{X}^{n, m}$ is an $O M$-mapping. Hence by 5.6 of [24, p. 33], $S C_{m}^{n}(f)$ is an $O M$-mapping.

To prove $(3) \Rightarrow(1)$, let $\left\{y_{r}\right\}_{r \in \mathbb{N}}$ be a sequence of points in $Y$ converging to a point $y \in Y$. Consider $\left\{z_{1}, \ldots, z_{n-1}\right\} \subset Y \backslash\left(\{y\} \cup\left\{y_{r}\right\}_{r \in \mathbb{N}}\right)$ such that $z_{i} \neq z_{j}$ if $i \neq j$. Let $M_{i}$ be a component of $f^{-1}\left(z_{i}\right)$, for each $i \in\{1, \ldots, n-1\}$ and let $M_{n}$ be a component of $f^{-1}(y)$. By Proposition 2.4, $\rho_{X}^{n, m}\left(\left\langle M_{1}, \ldots, M_{n}\right\rangle\right)$ is a component of $S C_{m}^{n}(f)^{-1}\left(\rho_{Y}^{n, m}\left(\left\{z_{1}, \ldots, z_{n-1}, y\right\}\right)\right)$. Clearly the sequence $\left\{\rho_{Y}^{n, m}\left(\left\{z_{1}, \ldots, z_{n-1}, y_{r}\right\}\right)\right\}_{r \in \mathbb{N}}$ converges to $\rho_{Y}^{n, m}\left(\left\{z_{1}, \ldots, z_{n-1}, y\right\}\right)$.

Since $S C_{m}^{n}(f)$ is an $O M$-mapping, by Lemma 6.2,

$$
\rho_{X}^{n, m}\left(\left\langle M_{1}, \ldots, M_{n}\right\rangle\right) \cap \limsup _{s \rightarrow \infty} S C_{m}^{n}(f)^{-1}\left(\rho_{Y}^{n, m}\left(\left\{z_{1}, \ldots, z_{n-1}, y_{s}\right\}\right)\right) \neq \emptyset \text {. }
$$


Let $A \in\left\langle M_{1}, \ldots, M_{n}\right\rangle$ such that

$$
\rho_{X}^{n, m}(A) \in \limsup _{s \rightarrow \infty} S C_{m}^{n}(f)^{-1}\left(\rho_{Y}^{n, m}\left(\left\{z_{1}, \ldots, z_{n-1}, y_{s}\right\}\right)\right) .
$$

Then, there exists a subsequence $\left\{\rho_{X}^{n, m}\left(A_{s_{k}}\right)\right\}_{k \in \mathbb{N}}$ such that for each positive integer $k, \rho_{X}^{n, m}\left(A_{s_{k}}\right) \in S C_{m}^{n}(f)^{-1}\left(\rho_{Y}^{n, m}\left(\left\{z_{1}, \ldots, z_{n-1}, y_{s_{k}}\right\}\right)\right)$ and

$$
\lim _{k \rightarrow \infty} \rho_{X}^{n, m}\left(A_{s_{k}}\right)=\rho_{X}^{n, m}(A) .
$$

Let $a \in A \cap M_{n}$. Since $\lim _{k \rightarrow \infty} A_{s_{k}}=A_{n}$, there exists a sequence $\left\{a_{s_{k}}\right\}_{k \in \mathbb{N}}$, with $a_{s_{k}} \in A_{s_{k}}$, such that converges to $a \in A$. Thus exists a positive integer $l_{0}$ such that $f\left(a_{s_{k}}\right)=y_{s_{k}}$ for each $k \geq l_{0}$. Hence

$$
a \in A \cap M_{n} \cap \limsup _{s \rightarrow \infty} f^{-1}\left(y_{s}\right) .
$$

Therefore, by Lemma $6.2, f$ is $O M$ mapping.

Clearly $(2) \Rightarrow(1)$.

Corollary 6.4. Let $f: X \rightarrow Y$ be a mapping between continua. Then the following conditions are equivalent.

(1) $f$ is an OM-mapping.

(2) $C_{2}(f)$ is an OM-mapping.

(3) $S C_{1}^{2}(f)$ is an OM-mapping.

Proof. It follows from [12, Theorem 14] and Theorem 6.3.

Theorem 6.5. Let $f: X \rightarrow Y$ be a mapping between continua and let $n \geq 3$. Then the following conditions are equivalent.

(1) $f$ is monotone.

(2) $C_{n}(f)$ is monotone.

(3) $C_{n}(f)$ is OM-mapping.

(4) $S C_{m}^{n}(f)$ is an OM-mapping, $2 \leq m \leq n-2$.

Proof. By Theorem 4.1, (1) and (2) are equivalent. (1) $\Rightarrow(3)$ follows from Theorem 4.1. By Theorem $6.3,(3) \Rightarrow(4)$. We will prove that $(4) \Rightarrow(1)$. Since $S C_{m}^{n}(f)$ is an $O M$-mapping, by $(4.9)$ of $\left[24\right.$, p. 16], $S C_{m}^{n}(f)$ is confluent. Thus, by Theorem $5.3, f$ is monotone.

The next example shows that (1) does not imply (3) in Theorem 6.3.

EXAMPLE 6.6. There exist continua $X$ and $Y$ and an $O M$-mapping $f$ : $X \rightarrow Y$ such that $S C_{m}^{n}(f)$ is not an $O M$-mapping.

Consider the mapping $f:[-1,1] \rightarrow[0,1]$ defined by $f(x)=|x|$. Clearly, $f$ is an $O M$-mapping but not monotone. By Theorem $6.5, S C_{m}^{n}(f)$ is not an $O M$-mapping for $n \geq 4$ and $2 \leq m \leq n-2$. 


\section{JOINING MAPPINGS}

Theorem 7.1. Let $f: X \rightarrow Y$ be a mapping between continua and let $n \geq 2$. Consider the following conditions:

(1) $f$ is joining;

(2) $C_{n}(f)$ is joining;

(3) $S C_{m}^{n}(f)$ is joining.

Then condition (2) implies (3) and each of the conditions (2) and (3) implies (1).

Proof. $(2) \Rightarrow(3)$. Since $\rho_{Y}^{n, m}$ is monotone and $C_{n}(f)$ is a joining mapping, $\rho_{Y}^{n, m} \circ C_{n}(f)$ is a joining mapping. Thus $S C_{m}^{n}(f) \circ \rho_{X}^{n, m}$ is a joining mapping. By (5.21) of [24, p. 33], $S C_{m}^{n}(f)$ is a joining mapping.

We will prove that $(3) \Rightarrow(1)$. Let $B$ be a proper subcontinuum of $Y$. Let $C_{1}$ and $C_{2}$ components of $f^{-1}(B)$. Consider $\left\{z_{1}, \ldots, z_{m}\right\} \subset Y \backslash B$ such that $z_{i} \neq z_{j}$ if $i \neq j$. For each $i \in\{1, \ldots, m\}$, let $M_{i}$ be a component of $f^{-1}\left(z_{i}\right)$. By Proposition $2.4 \rho_{X}^{n, m}\left(\left\langle M_{1}, \ldots, M_{m}, C_{i}\right\rangle\right)$ is be a component of $S C_{m}^{n}(f)^{-1}\left(\rho_{Y}^{n, m}\left(\left\langle\left\{z_{1}\right\}, \ldots,\left\{z_{m}\right\}, B\right\rangle\right)\right)$ for $i \in\{1,2\}$. Since $S C_{m}^{n}(f)$ is a joining mapping,

$S C_{m}^{n}(f)\left(\rho_{X}^{n, m}\left(\left\langle M_{1}, \ldots, M_{m}, C_{1}\right\rangle\right)\right) \cap S C_{m}^{n}(f)\left(\rho_{X}^{n, m}\left(\left\langle M_{1}, \ldots, M_{m}, C_{2}\right\rangle\right)\right) \neq \emptyset$.

So $C_{n}(f)\left(\left\langle M_{1}, \ldots, M_{m}, C_{1}\right\rangle\right) \cap C_{n}(f)\left(\left\langle M_{1}, \ldots, M_{m}, C_{2}\right\rangle\right) \neq \emptyset$. Thus $f\left(C_{1}\right) \cap$ $f\left(C_{2}\right) \neq \emptyset$.

Clearly $(2) \Rightarrow(1)$.

\section{Light MAPPINGS}

Theorem 8.1. Let $f: X \rightarrow Y$ be a mapping between continua and let $n \geq 2$. Consider the following conditions:

(1) $f$ is light;

(2) $C_{n}(f)$ is light;

(3) $S C_{m}^{n}(f)$ is light.

Then each of the conditions (2) and (3) implies (1).

Proof. $(2) \Rightarrow(1)$. It follows from $C(f)=\left.C_{n}(f)\right|_{C(X)}$ and $[6$, Theorem 3.10].

$(3) \Rightarrow(1)$. To prove that $f$ is a light mapping, suppose that exists $y \in Y$ such that $f^{-1}(y)$ is not totally disconnected. Let $M$ be a nondegenerate component of $f^{-1}(y)$. Consider $\left\{y_{1}, \ldots, y_{n-1}\right\} \subset Y \backslash\{y\}$ such that $y_{i} \neq y_{j}$ if $i \neq j$. Let $M_{i}$ be a component of $f^{-1}\left(y_{i}\right)$, for each $i \in\{1, \ldots, n-1\}$. By Proposition $2.4 \rho_{X}^{n, m}\left(\left\langle M_{1}, \ldots, M_{n-1}, M\right\rangle\right)$ is a subcontinuum nondegenerate of $S C_{m}^{n}(f)^{-1}\left(\rho_{Y}^{n, m}\left(\left\{y_{1}, \ldots, y_{n-1}, y\right\}\right)\right)$, a contradiction.

The next example shows that (1) does not imply (3) in Theorem 8.1. 
ExAmple 8.2. There exist continua $X$ and $Y$ and a light mapping $f$ : $X \rightarrow Y$ such that $S C_{m}^{n}(f): S C_{m}^{n}(X) \rightarrow S C_{m}^{n}(Y)$ is not light.

Let $\mathbb{C}$ be the complex plane and $S^{1} \subset \mathbb{C}$ the unit circle centered at the origin. Fix $n$ and $m$. Define $f: S^{1} \rightarrow S^{1}$ by $f(z)=z^{n}$. Clearly $f$ is a light mapping. Consider

$$
\mathcal{A}=\left\{\rho_{X}^{n, m}\left(\left\{e^{i \theta}, e^{i\left(\frac{2 \pi}{n}+\theta\right)}, \ldots, e^{i\left(\frac{2(n-1) \pi}{n}+\theta\right)}\right\}\right) \in S C_{m}^{n}(X): 0 \leq \theta \leq \frac{2 \pi}{n}\right\} .
$$

Notice that $\mathcal{A} \subset S C_{m}^{n}(f)^{-1}\left(\rho_{Y}^{n, m}\left(C_{m}\left(S^{1}\right)\right)\right)$. It is easy to show that $\mathcal{A}$ is an arc in $S C_{m}^{n}(X)$. Therefore $S C_{m}^{n}(f)$ is not a light mapping.

\section{ATOMIC MAPPINGS}

THEOREM 9.1. Let $f: X \rightarrow Y$ be a mapping between continua and $n \geq 2$. The conditions are equivalent.

(1) $f$ is homeomorphism.

(2) $C_{n}(f)$ is atomic.

(3) $S C_{m}^{n}(f)$ is atomic.

Proof. By Theorem 3.1, (1) implies (2) and (3).

$(2) \Rightarrow(1)$ is proved in [12, Theorem 43].

$(3) \Rightarrow(1)$ follows from Proposition 2.1 and $(6.3)$ of [24, p. 51].

\section{Atriodic MAPPINGS}

TheOREM 10.1. Let $f: X \rightarrow Y$ be a mapping between continua and $n \geq 2$. If $S C_{m}^{n}(f)$ is atriodic, then $f$ is atriodic.

Proof. Let $D$ be a proper subcontinuum of $Y$. Consider $\left\{z_{1}, \ldots, z_{n-1}\right\} \subset$ $Y \backslash D$ such that $z_{i} \neq z_{j}$ if $i \neq j$. Then there exist two components $\mathcal{D}_{1}$ and $\mathcal{D}_{2}$ of $S C_{m}^{n}(f)^{-1}\left(\rho_{Y}^{n, m}\left(\left\langle\left\{z_{1}\right\}, \ldots,\left\{z_{n-1}\right\}, D\right\rangle\right)\right)$ such that

1. $S C_{m}^{n}(f)\left(\mathcal{D}_{1}\right) \cup S C_{m}^{n}(f)\left(\mathcal{D}_{2}\right)=\rho_{Y}^{n, m}\left(\left\langle\left\{z_{1}\right\}, \ldots,\left\{z_{n-1}\right\}, D\right\rangle\right)$,

2. for each component $\mathcal{K}$ of $S C_{m}^{n}(f)^{-1}\left(\rho_{Y}^{n, m}\left(\left\langle\left\{z_{1}\right\}, \ldots,\left\{z_{n-1}\right\}, D\right\rangle\right)\right)$, we have either $S C_{m}^{n}(f)(\mathcal{K})=\rho_{Y}^{n, m}\left(\left\langle\left\{z_{1}\right\}, \ldots,\left\{z_{n-1}\right\}, D\right\rangle\right)$ or $S C_{m}^{n}(f)(\mathcal{K})$ $\subset S C_{m}^{n}(f)\left(\mathcal{D}_{1}\right)$ or $S C_{m}^{n}(f)(\mathcal{K}) \subset S C_{m}^{n}(f)\left(\mathcal{D}_{2}\right)$.

Notice that for each $j \in\{1,2\},\left(\rho_{X}^{n, m}\right)^{-1}\left(\mathcal{D}_{j}\right) \subset \mathcal{C}_{n-1}^{n}(X)$. So, we can find subsets $M_{1}^{j}, \ldots, M_{n}^{j}$ of $X$ such that for each $i \in\{1, \ldots, n-1\}, M_{i}^{j}$ is be a component of $f^{-1}\left(z_{i}\right), M_{n}^{j}$ is a component of $f^{-1}(D)$ and since $\mathcal{D}_{j}$ is a component of $S C_{m}^{n}(f)^{-1}\left(\rho_{Y}^{n, m}\left(\left\langle\left\{z_{1}\right\}, \ldots,\left\{z_{n-1}\right\}, D\right\rangle\right)\right)$ then $\mathcal{D}_{j} \cap \rho_{X}^{n, m}\left(\left\langle M_{1}^{j}, \ldots, M_{n}^{j}\right\rangle\right) \neq \emptyset$. It follows from Proposition 2.4 that $\mathcal{D}_{j}=\rho_{X}^{n, m}\left(\left\langle M_{1}^{j}, \ldots, M_{n}^{j}\right\rangle\right)$. Thus, by 1 ., $f\left(M_{n}^{1}\right) \cup f\left(M_{n}^{2}\right)=D$.

Now, let $K$ be a component of $f^{-1}(D)$. Since $\rho_{X}^{n, m}\left(\left\langle M_{1}^{1}, \ldots, M_{n-1}^{1}, K\right\rangle\right)$ is a component of $S C_{m}^{n}(f)^{-1}\left(\rho_{Y}^{n, m}\left(\left\langle\left\{y_{1}\right\}, \ldots,\left\{y_{n-1}\right\}, D\right\rangle\right)\right)$ by $2 ., f(K)=D$ or $f(K) \subset f\left(M_{n}^{1}\right)$ or $f(K) \subset f\left(M_{n}^{2}\right)$. 


\section{LOCALLY MAPPINGS}

Theorem 11.1. Let $f: X \rightarrow Y$ be a mapping between continua and let $n \geq 3$. If $S C_{m}^{n}(f)$ is a local homeomorphism then, $f$ is a homeomorphism.

Proof. It follows from (4.26) of [24, p. 20] and Theorem 3.2.

S. Nadler Jr. in [26, Lemma 2.3], defines a metric for a quotient space obtained identifying a subcontinuum to a point. In a similar way we are going to define a metric on the $S C_{m}^{n}(X)$. Let

$$
\Gamma_{n, m}=\left\{C_{m}(X) \cup\{A\}: A \in C_{n}(X)\right\} .
$$

Define $G_{n, m}: S C_{m}^{n}(X) \rightarrow \Gamma_{n, m}$ by

$$
G_{n, m}\left(\rho_{X}^{n, m}(A)\right)=C_{m}(X) \cup\left(\rho_{X}^{n, m}\right)^{-1}\left(\rho_{X}^{n, m}(A)\right) .
$$

Then $G_{n, m}$ is a homeomorphism. Next, define

$$
\sigma_{n, m}: S C_{m}^{n}(X) \times S C_{m}^{n}(X) \rightarrow[0, \infty)
$$

by

$$
\sigma_{n, m}\left(\rho_{X}^{n, m}(A), \rho_{X}^{n, m}(B)\right)=\mathcal{H}^{2}\left(G _ { n , m } \left(\rho_{X}^{n, m}(A), G_{n, m}\left(\rho_{X}^{n, m}(B)\right),\right.\right.
$$

where $\mathcal{H}^{2}$ is the Hausdorff metric induced by the Hausdorff metric $\mathcal{H}$ on $C_{n}(X)$. Thus $\sigma_{n, m}$ is a metric.

Lemma 11.2. Let $X$ be a continuum, $\varepsilon>0$ and $n \geq 2$. Let $A, B \in C_{n}(X)$ such that $\mathcal{H}(A, B)<\varepsilon$, then $\sigma_{n, m}\left(\rho_{X}^{n, m}(A), \rho_{X}^{n, m}(B)\right)<\varepsilon$.

Proof. Let $A, B \in C_{n}(X)$ such that $\mathcal{H}(A, B)<\varepsilon$, then $\{B\} \in \mathcal{V}_{\varepsilon}^{\mathcal{H}}(\{A\})$ and $\{A\} \in \mathcal{V}_{\varepsilon}^{\mathcal{H}}(\{B\})$. Notice that $G_{n, m}\left(\rho_{X}^{n, m}(A)\right) \subset \mathcal{V}_{\varepsilon}^{\mathcal{H}}\left(G_{n, m}\left(\rho_{X}^{n, m}(B)\right)\right)$ and $G_{n, m}\left(\rho_{X}^{n, m}(B)\right) \subset \mathcal{V}_{\varepsilon}^{\mathcal{H}}\left(G_{n, m}\left(\rho_{X}^{n, m}(A)\right)\right)$. Thus

$$
\mathcal{H}^{2}\left(G_{n, m}\left(\rho_{X}^{n, m}(A)\right), G_{n, m}\left(\rho_{X}^{n, m}(B)\right)\right)<\varepsilon .
$$

Lemma 11.3. Let $X$ be a continuum, $\varepsilon>0$ and $n \geq 2$. Take $A \in C(X)$ such that $\operatorname{diam}(A)<\varepsilon$ and let $\left\{z_{1}, \ldots, z_{m}\right\} \subset X \backslash A$ such that $z_{i} \neq z_{j}$ if $i \neq j$. Then $\operatorname{diam}\left(\rho_{X}^{n, m}\left(\left\langle\left\{z_{1}\right\}, \ldots,\left\{z_{m}\right\}, A\right\rangle\right)<\varepsilon\right.$.

Proof. Let $B, D \in\left\langle\left\{z_{1}\right\}, \ldots,\left\{z_{m}\right\}, A\right\rangle$. Consider $D_{1}=D \cap A$ and $B_{1}=B \cap A$. Notice that $d(x, c)<\operatorname{diam}(A)$ for each $c \in D_{1}$ and for each $x \in$ $B_{1}$. Then $B \subset \mathcal{V}_{\varepsilon}^{d}(D)$ and $D \subset \mathcal{V}_{\varepsilon}^{d}(B)$. Thus $\mathcal{H}(B, C)<\varepsilon$. By Lemma 11.2, $\sigma_{n, m}\left(\rho_{X}^{n, m}(B), \rho_{X}^{n, m}(D)\right)<\varepsilon$. Therefore $\operatorname{diam}\left(\rho_{X}^{n, m}\left(\left\langle\left\{z_{1}\right\}, \ldots,\left\{z_{m}\right\}, A\right\rangle\right)\right)<\varepsilon$.

THEOREM 11.4. Let $f: X \rightarrow Y$ be a mapping between continua and $n \geq 2$. If $S C_{m}^{n}(f)$ is locally confluent then, $f$ is locally confluent. 
Proof. By [24, Theorem 4.38], there exists $\varepsilon>0$ such that for each subcontinuum $\mathcal{A}$ of $S C_{m}^{n}(Y)$ of diameter less than $\varepsilon$ and each component $\mathcal{D}$ of $S C_{m}^{n}(f)^{-1}(\mathcal{A})$ is mapped by $S C_{m}^{n}(f)$ onto $\mathcal{A}$. Let $B$ be a subcontinuum of $Y$ of diameter less that $\varepsilon$ and $D$ be a component of $f^{-1}(B)$. Consider $\left\{z_{1}, \ldots, z_{m}\right\} \subset Y \backslash B$ such that $z_{i} \neq z_{j}$ if $i \neq j$. Let $M_{i}$ be a component of $f^{-1}\left(z_{i}\right)$, for each $i \in\{1, \ldots, m\}$. By Lemma 11.3, $\rho_{Y}^{n, m}\left(\left\langle\left\{z_{1}\right\}, \ldots,\left\{z_{m}\right\}, B\right\rangle\right)$ has diameter less that $\varepsilon$. By Proposition 2.4, $\rho_{X}^{n, m}\left(\left\langle M_{1}, \ldots, M_{m}, D\right\rangle\right)$ is a component of $S C_{m}^{n}(f)^{-1}\left(\rho_{Y}^{n, m}\left(\left\langle\left\{z_{1}\right\}, \ldots,\left\{z_{m}\right\}, B\right\rangle\right)\right)$. Thus

$$
S C_{m}^{n}(f)\left(\rho_{X}^{n, m}\left(\left\langle M_{1}, \ldots, M_{m}, D\right\rangle\right)=\left(\rho_{Y}^{n, m}\left\langle\left\{z_{1}\right\}, \ldots,\left\{z_{m}\right\}, B\right\rangle\right) .\right.
$$

We conclude that $f(D)=B$. By [24, Theorem 4.38], $f$ is locally confluent.

THEOREM 11.5. Let $f: X \rightarrow Y$ be a mapping between continua and $n \geq$ 2. If $S C_{m}^{n}(f)$ is locally weakly confluent then, $f$ is locally weakly confluent.

Proof. By 4.37 of $[24$, p. 23], there exists $\varepsilon>0$ such that for each subcontinuum $\mathcal{A}$ of $S C_{m}^{n}(Y)$ of diameter less than $\varepsilon$, there exists a component $\mathcal{D}$ of $S C_{m}^{n}(f)^{-1}(\mathcal{A})$ such that $S C_{m}^{n}(f)(\mathcal{D})=\mathcal{A}$. Let $B$ be a subcontinuum of $Y$ of diameter less than $\varepsilon$. Consider $\left\{z_{1}, \ldots, z_{n-1}\right\} \subset Y \backslash B$ such that $z_{i} \neq z_{j}$ if $i \neq j$. By Lemma 11.3, $\rho_{Y}^{n, m}\left(\left\langle\left\{z_{1}\right\}, \ldots,\left\{z_{n-1}\right\}, B\right\rangle\right)$ has diameter less than $\varepsilon$. Since $S C_{m}^{n}(f)$ is a locally weakly confluent mapping, there exists a component $\mathcal{B}$ of $S C_{m}^{n}(f)^{-1}\left(\rho_{Y}^{n, m}\left(\left\langle\left\{z_{1}\right\}, \ldots,\left\{z_{n-1}\right\}, B\right\rangle\right)\right)$ such that $S C_{m}^{n}(f)(\mathcal{B})=\rho_{Y}^{n, m}\left(\left\langle\left\{z_{1}\right\}, \ldots,\left\{z_{n-1}\right\}, B\right\rangle\right)$. Notice that $\left(\rho_{Y}^{n, m}\right)^{-1}(\mathcal{B}) \subset$ $\mathcal{C}_{n-1}^{n}(X)$. Thus, we can find subset $M_{1}, \ldots, M_{n}$ of $X$, such that for each $i \in\{1, \ldots, n-1\}, M_{i}$ is a component of $f^{-1}\left(z_{i}\right), M_{n}$ is a component of $f^{-1}(B)$ and $\rho_{X}^{n, m}\left(\left\langle M_{1}, \ldots, M_{n}\right\rangle\right) \cap \mathcal{C} \neq \emptyset$. It follows from Proposition 2.4 that $\rho_{X}^{n, m}\left(\left\langle M_{1}, \ldots, M_{n}\right\rangle\right)$ is a component of

$$
S C_{m}^{n}(f)^{-1}\left(\rho_{Y}^{n, m}\left(\left\langle\left\{z_{1}\right\}, \ldots,\left\{z_{n-1}\right\}, B\right\rangle\right)\right) .
$$

Hence $\mathcal{B}=\rho_{X}^{n, m}\left(\left\langle M_{1}, \ldots, M_{n}\right\rangle\right)$. Therefore $f\left(M_{n}\right)=B$. By $[24$, Theorem 4.37], $f$ is locally weakly confluent.

\section{Problems}

(1) Is there weakly confluent mapping $f: X \rightarrow Y$ such that either $C_{n}(f)$ is not weakly confluent or $S C_{m}^{n}(f)$ is not weakly confluent?

(2) Is there hereditarily weakly confluent mapping $f: X \rightarrow Y$ such that either $C_{n}(f)$ is not hereditarily weakly confluent or $S C_{m}^{n}(f)$ is not hereditarily weakly confluent?

(3) Is there semi-confluent mapping $f: X \rightarrow Y$ such that either $C_{n}(f)$ is not semi-confluent or $S C_{m}^{n}(f)$ is not semi-confluent?

(4) Is there $O M$-mapping $f: X \rightarrow Y$ such that either $C_{n}(f)$ is not $O M$ or $S C_{m}^{n}(f)$ is not $O M$ for $n>2$ ? 
(5) Is there joining mapping $f: X \rightarrow Y$ such that either $C_{n}(f)$ is not joining or $S C_{m}^{n}(f)$ is not joining?

ACKNOWLEDGEMENTS.

The authors wish to thank to the Universidad Autónoma del Estado de México for its financial support through the research project "Funciones inducidas entre continuos y funciones inducidas entre n-ésimo hiperespacio de continuos", 3704/2014/CID.

\section{REFERENCES}

[1] J. Camargo, Openness of the induced map $C_{n}(f)$, Bol. Mat. (N.S.) 16 (2009), 115123.

[2] J. Camargo and S. Macías, On strongly freely decomposable and induced maps, Glas. Mat. Ser. III 48 (2013), 429-442.

[3] F. Barragán, Induced maps on n-fold symmetric product suspensions, Topology Appl. 158 (2011), 1192-1205.

[4] J. J. Charatonik, Recent results on induced mappings between hyperspaces of continua, Topology. Proc. 22 (1997), 103-122.

[5] J. J. Charatonik and W. J. Charatonik, Hereditarily weakly confluent induced mappings are homeomorphisms, Colloq. Math. 75 (1998), 195-203.

[6] J. J. Charatonik and W. J. Charatonik, Lightness of induced mappings, Tsukuba J. Math. 22 (1998), 179-192.

[7] J. J. Charatonik and W. J. Charatonik, Induced MO-mappings, Tsukuba J. Math. 23 (1999), 245-252.

[8] J. J. Charatonik and W. J. Charatonik, Limit properties of induced mappings, Topology Appl. 100 (2000), 103-118.

[9] J.J. Charatonik and W.J. Charatonik, Inverse limits and openness of the induced mappings, Topology Appl. 114 (2001), 235-260.

[10] J. J. Charatonik and W. J. Charatonik Semi-confluent mappings, Math. Pannon. 12 (2001), 39-54.

[11] J. J. Charatonik, W. J. Charatonik and A. Illanes, Openness of induced mappings, Topology Appl. 98 (1999), 67-80.

[12] J. J. Charatonik, A. Illanes and S. Macías, Induced mappings on the hyperspaces $C_{n}(X)$ of a continuum $X$, Houston J. Math. 28 (2002), 781-805.

[13] W. J. Charatonik, Arc approximation property and confluence of induced mappings, Rocky Mountain J. Math. 28 (1998), 107-154.

[14] W. J. Charatonik, Openness and monotoneity of induced mappings, Proc. Amer. Math. Soc. 127 (1999), 3729-3731.

[15] J. Dugundji, Topology, Allyn and Bacon, Inc., Boston, 1966.

[16] G. Higuera and A. Illanes, Induced mappings on symmetric products, Topology Proc. 37 (2011), 367-401.

[17] H. Hosokawa, Induced mappings between hyperspaces, Bull. Tokyo Gakugei Univ. (4) 41 (1989), 1-6.

[18] H. Hosokawa, Mappings of hyperspaces induced by refinable mappings, Bull. Tokyo Gakugei Univ. (4) 42 (1990), 1-8.

[19] H. Hosokawa, Induced mappings between hyperspaces. II, Bull. Tokyo Gakugei Univ. (4) 44 (1992), 1-7.

[20] H. Hosokawa, Induced mappings on hyperspaces, Tsukuba J. Math. 21 (1997), 239 259. 
[21] H. Hosokawa, Induced mappings on hyperspaces. II, Tsukuba J. Math. 21 (1997), $773-783$.

[22] A. Illanes and S. B. Nadler, Jr., Hyperspaces. Fundamentals and recent advances, Marcel Dekker, New York, 1999.

[23] S. Macías, On the hyperspaces $C_{n}(X)$ of a continuum X, Topology Appl. 109 (2001), $237-256$.

[24] T. Maćkowiak, Continuous mappings on continua, Dissertationes Math. (Rozprawy Mat.) 158 (1979), 95 pp.

[25] T. Maćkowiak, Singular arc-like continua, Dissertationes Math. (Rozprawy Mat.) 257 (1986), 40 pp.

[26] S. B. Nadler, Jr., A fixed point theorem for hyperspace suspensions, Houston J. Math. 5 (1979), 125-132.

[27] S. B. Nadler, Jr., Hyperspaces of Sets, Marcel Dekker, New York, 1978.

[28] S. B. Nadler Jr., Continuum theory. An introduction, Marcel Dekker, New York, 1992.

\section{J. G. Anaya}

Universidad Autónoma del Estado de México, Facultad de Ciencias, Instituto Literario No. 100, Col. Centro, C. P. 50000,

Toluca, Estado de México

México.

E-mail: jgao@uaemex.mx

F. Capulín

Universidad Autónoma del Estado de México, Facultad de Ciencias, Instituto Literario No. 100, Col. Centro, C. P. 50000

Toluca, Estado de México

México.

E-mail: fcapulin@gmail.com

F. Orozco

Universidad Autónoma del Estado de México, Facultad de Ciencias, Instituto Literario No. 100, Col. Centro, C. P. 50000,

Toluca, Estado de México

México.

E-mail: forozcozitli@gmail.com

M.A. Lara

Universidad Autónoma del Estado de México, Facultad de Ciencias, Instituto Literario No. 100, Col. Centro, C. P. 50000,

Toluca, Estado de México

México.

E-mail: nanoji@live.com.mx

Received: 8.5.2015.

Revised: 13.2 .2016 \& 22.6.2016 